\title{
A Novel Metrics to Predict Right Heart Failure After Left Ventricular Assist Device Implantation
}

Federica Valente ( $\nabla$ valentefederica91@gmail.com )

Erasme Hospital: Hopital Erasme

\section{Constantin Stefanidis}

Erasme Hospital: Hopital Erasme

Jean-Luc Vachiéry

Erasme Hospital: Hopital Erasme

Céline Dewachter

Erasme Hospital: Hopital Erasme

\section{Edgard Engelman}

Erasme Hospital: Hopital Erasme

Frédéric Vanden Eynden

Erasme Hospital: Hopital Erasme

Ana Roussoulières

Erasme Hospital: Hopital Erasme

Research

Keywords: Advanced Heart Failure, Mechanical circulatory support, Right heart failure, Prediction models

Posted Date: September 30th, 2021

DOI: https://doi.org/10.21203/rs.3.rs-934177/v1

License: (c) (1) This work is licensed under a Creative Commons Attribution 4.0 International License.

Read Full License 


\section{Abstract}

\section{Background}

Right Heart Failure (RHF) is a severe complication that can occur after left ventricular assist device (LVAD) implantation, increasing early and late mortality. Although numerous RHF predictive scores have been developed, limited data exist on the external validation of these models.

We therefore aimed at comparing existent risk score models and identifying predictors of severe RHF at our center.

\section{Methods}

In this retrospective, single center analysis, clinical, biological and functional data were collected in patients implanted with a LVAD between 2011 and 2020. Early severe RHF was defined as use of inotropes for $\geq 14$ days, nitric oxide use for $\geq 48$ hours or unplanned right-sided circulatory support.

Risk models were evaluated for the primary outcome of RHF or RVAD implantation by means of logistic regression and receiver operating characteristic curves.

\section{Results}

Among 92 patients implanted, 24 (26\%) developed early severe RHF. The EUROMACS-RHF risk score performed the best in predicting RHF (C=0.82 - 95\% Cl: 0.68-0.90), compared with the other scores (Michigan, CRITT).

In addition, we developed a new model, based on four variables selected for the best reduced logistic model: the INTERMACS level, the number of inotropes used, the ratio of right atrial/pulmonary capillary wedge pressure and the ratio of right ventricle/left ventricle diameters by echocardiography. This model demonstrated significant discrimination of RHF (C=0.91 - 95\% Cl: 0.76-0.96).

\section{Conclusion}

Amongst available risk scores, EUROMACS-RHF performs best to predict the occurrence of RHF after LVAD implantation. Our model's performance compares well to the EUROMACS-RHF score, adding a more objective parameter to RV function evaluation.

\section{Introduction}

Heart transplantation (HT) is the gold-standard therapy for end-stage heart failure (HF) but represents a limited therapeutic option ${ }^{1,2}$.

The increasing number of patients with refractory, advanced HF and the declining willingness for organ donation have resulted in expanded waiting lists, increased waiting times and mortality in HT waiting list 
patients. ${ }^{3-5}$.

At present, implantable left ventricular assist devices (LVADs) represent an available and effective alternative to $\mathrm{HT}$, allowing a decrease in morbidity and mortality observed in advanced HF, particularly in patients on HT waiting lists ${ }^{6,7}$.

Despite the widespread adoption and success of LVADs used for bridge-to-transplant (BTT) or destination-therapy (DT) indications, complications are numerous limiting its efficacy ${ }^{8,9}$.

If the prevention and management of certain complications, such as major bleeding, pump thrombosis, neurological accidents and infections have improved, especially with new generation pumps, postoperative right heart failure (RHF) remains nevertheless a perennial issue to LVAD success.

Post-LVAD acute RHF is common and prevalence has been reported between $4 \%$ and $50 \%{ }^{10-15}$. It is characterized by the Interagency Registry for Mechanically Assisted Circulatory Support (INTERMACS) ${ }^{16}$ as documented elevations of central venous pressure associated with more than one clinical or biological manifestations ${ }^{16}$. Severe acute RHF is described by INTERMACS and most clinical investigators as the need for prolonged post- implant inotropes, inhaled nitric oxide (NO) or intravenous vasodilators for more than 14 days after LVAD implant, or the requirement for RV mechanical support ${ }^{16,17}$.

Severe RHF after LVAD implantation is associated with increased peri-operative mortality, prolonged length of stay, and decreased survival even after HT 13,15,18,19.

The pathophysiology of RHF after LVAD implant is complex and heterogeneous, making it difficult to establish a stratification or risk score ${ }^{19}$. Some risk scoring systems have been described to predict postLVAD RHF ${ }^{14,20-25}$. However, these studies are limited by the analysis of results in small cohorts, by their monocentric characteristics and by the heterogeneous nature of the LVADs.

The aim of this study was to perform a comparative analysis of commonly used RHF predictive risk models and to identify the best predictors of severe RHF in our cohort of patients undergoing primary LVAD implants.

\section{Methods}

\section{Study design}

This is a single center retrospective study using prospectively collected data from February 2011 to February 2020. All patients underwent LVAD implantations were included.

The devices used were all 2nd and 3rd generation continuous flow pumps: InCor (Berlin Heart, Berlin, Germany), Heartmate II (St-Jude Medical, USA), Heart Ware HVAD (Medtronic, USA), Heartmate 3 (Abbott 
Laboratories, USA). The indication of LVAD implantation was bridge-to-transplantation or bridge-todecision in all cases.

A retrospective analysis of pre-operative clinical, echocardiographic, laboratory, and hemodynamic data was performed to determine risk for $\mathrm{RH}$ failure after LVAD implantation.

Early (<30 days) severe RHF was defined as: receiving short- or long-term right-sided circulatory support (via an RV assist device or extracorporeal membrane oxygenator), continuous inotropic support for $\geq 14$ days, or nitric oxide ventilation for $\geq 48$ hours $^{17}$.

All data was obtained from electronic patient records and the present study was approved by the institutional review board.

\section{Variables collected}

Clinical data included demographics, heart failure etiology, medical and surgical history, preoperative treatment, INTERMACS class, need for life support including mechanical ventilation, renal replacement therapy, temporary mechanical circulatory support (MCS), requirement for a continuous intravenous inotrope (milrinone, dobutamine, or levosimendan) or vasopressor (norepinephrine, vasopressin) therapies.

Pre-operative laboratory data was obtained $<24 \mathrm{~h}$ before LVAD implantation and included a complete blood count, liver enzymes, renal function, and coagulation parameters.

Echocardiographic measurements closest to the time of LVAD implantation were recorded and included left ventricular ejection fraction (LVEF) and dimensions, the presence of visual right ventricular (RV) dysfunction (none, mild-moderate, severe), tricuspid regurgitation (none, mild, moderate, severe), values of tricuspid annular plane systolic excursion (TAPSE), S' wave, inferior vena cava diameter, estimation of systolic pulmonary arterial pressure (PAPs) and the ratio of RV/left ventricular (LV) diameter.

Hemodynamic variables were collected during the right heart catheterization, performed closest to the time of LVAD implantation. We included measurements of heart rate, systolic and diastolic pressure, right atrial pressure (RAP), pulmonary artery pressure (PAP), and pulmonary capillary wedge pressure (PCWP). Cardiac output was assessed by thermodilution. Systemic vascular resistance, transpulmonary gradient, pulmonary vascular resistance (PVR), RV stroke work index (RVSWI), pulmonary artery pulsatility index (PAPi) and right atrial to pulmonary capillary wedge pressure ratio (RAP/PCWP) were calculated 27,28. Samples of venous blood by the distal end of the pulmonary arterial catheter were collected to measure the mixed venous oxygen saturation (SvO2).

\section{Right ventricular failure predictive risk scores}

We applied all the predictive risk scores described in the literature and selected the EUROMACS-RHF risk score, the Michigan score and the CRITT score (Table 1) ${ }^{14,21,23}$ to our population. 
Table 1

Established Models for Right Heart Failure Prediction in LVAD Patients

\begin{tabular}{|c|c|c|c|c|c|c|}
\hline Study & $\begin{array}{l}\text { Study } \\
\text { design }\end{array}$ & $\begin{array}{l}\text { Patient } \\
\text { population }\end{array}$ & $\begin{array}{l}\text { RHF } \\
\text { rate } \\
(\mathrm{n} / \%)\end{array}$ & $\begin{array}{l}\text { C- } \\
\text { statistics }\end{array}$ & RHF definition & $\begin{array}{l}\text { Score } \\
\text { components } \\
\text { (points) }\end{array}$ \\
\hline \multirow[t]{4}{*}{$\begin{array}{l}\text { Michigan } \\
\text { RVF risk } \\
\text { score (2008) }\end{array}$} & $\begin{array}{l}P C \\
(1996- \\
2006)\end{array}$ & \multirow[t]{4}{*}{197} & \multirow[t]{4}{*}{$\begin{array}{l}68 \\
(35 \%)\end{array}$} & \multirow[t]{4}{*}{0.73} & \multirow{4}{*}{$\begin{array}{l}\text { Post-op } \\
\text { inotropes > } 14 \\
\text { days; iNO > 48 } \\
\text { h, or RVAD, or } \\
\text { hospital } \\
\text { discharge with } \\
\text { an } \\
\text { intravenous } \\
\text { inotrope }\end{array}$} & $\begin{array}{l}\text { - Vasopressor } \\
\text { requirement (4) }\end{array}$ \\
\hline & \multirow[t]{3}{*}{ USA } & & & & & $\begin{array}{l}\cdot \text { AST } \geq 80 \mathrm{IU} / \mathrm{I} \\
(2)\end{array}$ \\
\hline & & & & & & $\begin{array}{l}\cdot \text { Bilirubin } \geq 2.0 \\
\mathrm{mg} / \mathrm{dl}(2.5)\end{array}$ \\
\hline & & & & & & $\begin{array}{l}\text { - Creatinine } \geq 2.3 \\
\mathrm{mg} / \mathrm{dl}(3)\end{array}$ \\
\hline \multirow[t]{5}{*}{$\begin{array}{l}\text { CRITT score } \\
(2013)\end{array}$} & \multirow{5}{*}{$\begin{array}{l}\text { RCC } \\
(2003- \\
2011) \\
\text { USA }\end{array}$} & \multirow[t]{5}{*}{196} & \multirow[t]{5}{*}{$\begin{array}{l}45 \\
(23 \%)\end{array}$} & \multirow[t]{5}{*}{0.80} & \multirow{5}{*}{$\begin{array}{l}\text { RVAD implant. } \\
\text { Both pre-op } \\
\text { and post-op } \\
\text { BiVAD patients } \\
\text { were included }\end{array}$} & $\begin{array}{l}\text { - Severe RV } \\
\text { dysfunction (1) }\end{array}$ \\
\hline & & & & & & - Severe TR (1) \\
\hline & & & & & & $\begin{array}{l}\text { - Pre-operative } \\
\text { mechanical } \\
\text { ventilation (1) }\end{array}$ \\
\hline & & & & & & $\begin{array}{l}\cdot \text { CVP }>15 \text { mmhg } \\
\text { (1) }\end{array}$ \\
\hline & & & & & & $\begin{array}{l}\cdot \mathrm{HR}>100 / \mathrm{min} \\
(1)\end{array}$ \\
\hline \multirow{6}{*}{$\begin{array}{l}\text { EUROMACS- } \\
\text { RHF risk } \\
\text { score (2018) }\end{array}$} & $\begin{array}{l}P C \\
(2006- \\
2017)\end{array}$ & \multirow[t]{6}{*}{2000} & \multirow[t]{6}{*}{$\begin{array}{l}433 \\
(21.7 \%)\end{array}$} & \multirow[t]{6}{*}{0.70} & \multirow{6}{*}{$\begin{array}{l}\text { Post-op } \\
\text { inotropes > } 14 \\
\text { days; iNO > 48 } \\
\text { h, or RVAD }\end{array}$} & $\begin{array}{l}\text { - INTERMACS } \\
\text { class } 1-3(2)\end{array}$ \\
\hline & \multirow{5}{*}{ EU } & & & & & - Use of multiple \\
\hline & & & & & & $\begin{array}{l}\text { - Severe RV } \\
\text { dysfunction on }\end{array}$ \\
\hline & & & & & & $\begin{array}{l}\text { echocardiography } \\
\text { (2) }\end{array}$ \\
\hline & & & & & & $\begin{array}{l}\cdot \text { RAP/PCWP > } \\
0.54(2)\end{array}$ \\
\hline & & & & & & $\begin{array}{l}\cdot \text { Hemoglobin }<10 \\
\text { (1) }\end{array}$ \\
\hline $\begin{array}{l}\text { AST, aspartat } \\
\text { continuous flc } \\
\text { HR, heart rate } \\
\text { pre-operative; } \\
\text { failure; RV, rig }\end{array}$ & ansam & $\begin{array}{l}\text { e; ALT, alar } \\
\text { ular assist } \\
\text { ve cohort; } \\
\text { nary capilla } \\
\text { RVAD, righ }\end{array}$ & $\begin{array}{l}\text { e transa } \\
\text { jice; CV } \\
\text { C, retros } \\
\text { wedge }\end{array}$ & $\begin{array}{l}\text { ase; BiVA } \\
\text { ntral ven } \\
\text { tive case- } \\
\text { sure; RAP }\end{array}$ & $\begin{array}{l}\text { biventricular as } \\
\text { s pressure; iNO, } \\
\text { ontrol; post-op, } \\
\text { ght atrial press } \\
\text { TR, tricuspid re }\end{array}$ & $\begin{array}{l}\text { t device; CF-LVAD, } \\
\text { laled nitric oxide; } \\
\text { st-operative; pre-op, } \\
\text { RHF, right heart } \\
\text { gitation. }\end{array}$ \\
\hline
\end{tabular}


We excluded the UTAH RVF risk score ${ }^{20}$ as one of the score components was destination therapy (DT) not used in our population and the Pittsburgh decision tree, because of missing data.

These three risk models (Michigan, EUROMACS-RHF, CRITT) were evaluated for the primary outcome of RHF or RVAD implantation by means of logistic regression and receiver operating characteristic curves from their respective scores in our population.

\section{Statistical analysis}

The data are given as a number (\%) or as a mean \pm SD and median score with interquartile ranges.

For the description of preoperative variables, categorical data were compared by Chi-square test and continuous non longitudinal variables were compared by a Mann-Whitney test.

A logistic regression model was fitted for right ventricular failure, defined according to EUROMACS as receiving short- or long-term right-sided circulatory support, or continuous inotropic support for $\geq 14$ days, or NO administration for $\geq 48$ hours.

For inclusion in the logistic regression model, analysis of individual variables was performed by a likelihood ratio chi-square test for the categorical data test and by fitting a univariate logistic regression model to obtain a likelihood ratio test for continuous variables.

All variables with a p-value $<0.25$ were included to fit an initial full logistic regression model.

Given the small absolute numbers of occurrences ( 45 ventricular failures and 24 severe right ventricular failures), we searched for the best reduced model that would not include more than 4 variables for right ventricular failure, and might not differ by a $p$-value $<0.05$ calculated from a chi-square distribution for the value of the difference between the log-likelihoods of the full and the reduced logistic models. The reduced logistic models were generated by a hierarchical backward selection with switching.

Risk models (EUROMACS-RHF risk score, the Michigan score and the CRITT score) were evaluated for the primary outcome of RH failure or RVAD placement by means of logistic regression and receiver operating characteristic curves from their respective scores in our population.

All analyses were performed using the NCSS 20.0.2 statistical package (NCSS, LLC; Kaysville, UT).

\section{Results}

\section{Baseline characteristics and RHF incidence}

Ninety-two LVAD patients implanted from February 2011 to February 2020 were reviewed.

Of these patients, 70 had complete hemodynamic and echocardiographic data and composed the final cohort. 
Implantation strategy was BTT in $96 \%$ of patients and bridge to decision (BTD) in 4\%; no pumps were implanted as DT. Most LVAD implanted were represented by Heart Ware HVAD $(n=86,94 \%)$ followed by HeartMate II $(n=2,2 \%)$, HeartMate $3(n=2,2 \%)$ and Incor $(n=2,2 \%)$.

RHF was identified in 24 patients (26\%), of which 15 (62,5\%) were treated with inotropes for $\geq 14$ days, 8 (33\%) needed postoperatively right ventricular mechanical support, and 14 (58\%) were treated with inhaled pulmonary vasodilators for $\geq 48$ hours.

Baseline characteristics and preoperative predictors are presented in Table 2. 
Table 2

Baseline Demographics, Preoperative Comorbidities and Preoperative Right Heart Failure Predictors

\begin{tabular}{|c|c|c|c|}
\hline Variables & $\begin{array}{l}\text { No severe RV failure } \\
(n=68)\end{array}$ & $\begin{array}{l}\text { Severe RV failure } \\
(n=24)\end{array}$ & P-value \\
\hline Age $(y)$ & $\begin{array}{l}48.3 \pm 14.1-52[36.3- \\
60]\end{array}$ & $\begin{array}{l}51.1 \pm 12.9-53.5[44.3- \\
61]\end{array}$ & 0.48 \\
\hline Male/female & $51 / 17(75 \%$ / 25\%) & 17 / 7 (70.8\% / 29.2\%) & 0.59 \\
\hline Body mass index $\left(\mathrm{kg} / \mathrm{m}^{2}\right)$ & $\begin{array}{l}25.6 \pm 4.7-25.2[22.3- \\
29.1]\end{array}$ & $\begin{array}{l}25.9 \pm 5.4-25.7[24.1- \\
27.2]\end{array}$ & 0.94 \\
\hline \multicolumn{4}{|l|}{ Preoperative Comorbidities } \\
\hline Diabetes $(\mathrm{Y} / \mathrm{N})$ & $\begin{array}{l}22 / 44(33.3 \% / \\
66.7 \%)\end{array}$ & 4 / 18 (18.2\% / 81.8\%) & 0.17 \\
\hline Dyslipidemia $(\mathrm{Y} / \mathrm{N})$ & $\begin{array}{l}38 / 25(60.3 \% / \\
39.7 \%)\end{array}$ & 15 / $5(75 \% / 25 \%)$ & 0.23 \\
\hline Hypertension $(\mathrm{Y} / \mathrm{N})$ & $\begin{array}{l}32 / 34(48.5 \% / \\
51.5 \%)\end{array}$ & 15 / 7 (68.2\% / 31.8\%) & 0.10 \\
\hline Smoker & & & 0.56 \\
\hline No & $24(40.7 \%)$ & $8(38.1 \%)$ & \\
\hline Active & $22(37.3 \%)$ & $6(28.6 \%)$ & \\
\hline Previous & $13(22.0 \%)$ & $7(33.3 \%)$ & \\
\hline Illicit drugs $(\mathrm{Y} / \mathrm{N})$ & $5 / 50(9.1 \%$ / 90.9\%) & $0 / 11(0 \%$ / 100\%) & 0.29 \\
\hline Alcohol $(\mathrm{Y} / \mathrm{N})$ & $\begin{array}{l}18 / 39(31.6 \% / \\
68.4 \%)\end{array}$ & 4 / 12 (25\% / 75\%) & 0.61 \\
\hline $\begin{array}{l}\text { Chronic obstructive pulmonary } \\
\text { disease }(Y / N)\end{array}$ & $\begin{array}{l}10 / 42(19.2 \% / \\
80.8 \%)\end{array}$ & 5 / 12 (29.4\% / 70.6\%) & 0.38 \\
\hline
\end{tabular}

Values are represented as number (\%), or mean \pm SD - median [interquartile range]

P-values: $\mathrm{Chi}^{2}$ test or Mann-Whitney test $\mathrm{p}$-values

INTERMACS, interagency registry for mechanically assisted circulatory support; BMI, body mass index, ECMO, extracorporeal membrane oxygenation; IABP, intra-aortic balloon pump; AST, aspartate transaminase; ALT, alanine transaminase; INR, international normalized ratio; LVEF, left ventricular ejection fraction; TAPSE, tricuspid annular plane systolic excursion; HR, heart rate; SBP, systolic blood pressure; DBP, diastolic blood pressure; MAP, mean atrial pressure; RAP, right atrial pressure; PAPs, pulmonary artery systolic pressure; PAPd, pulmonary artery diastolic pressure; PAPm, pulmonary artery mean pressure; PVR, pulmonary vascular resistance; PCWP, pulmonary capillary wedge pressure; TPG, transpulmonary gradient; DPG, diastolic pulmonary gradient; $\mathrm{CO}$, cardiac output; $\mathrm{Cl}$, cardiac index; SV, stroke volume; SI, stroke index; PAPi, pulmonary artery pulsatility index; RVSWI, right ventricular stroke work index; Cpa, compliance pulmonary artery. 


\begin{tabular}{|llll|}
\hline Variables & $\begin{array}{l}\text { No severe RV failure } \\
(\mathbf{n}=68)\end{array}$ & $\begin{array}{l}\text { Severe RV failure } \\
(\mathbf{n = 2 4 )}\end{array}$ & P-value \\
\hline Atrial fibrillation $(\mathrm{Y} / \mathrm{N})$ & $\begin{array}{l}26 / 35(42.6 \% / \\
57.4 \%)\end{array}$ & $14 / 7(66.7 \% / 33.3 \%)$ & 0.057 \\
\hline Prior cardiac surgery $(\mathrm{Y} / \mathrm{N})$ & $\begin{array}{l}12 / 52(18.8 \% / \\
81.2 \%)\end{array}$ & $9 / 11(45 \% / 55 \%)$ & 0.018 \\
\hline Type of cardiopathy & & & 0.157 \\
\hline Idiopathic/UNK & $6(8.82 \%)$ & $0(0.00 \%)$ & \\
\hline Ischemic & $24(35.29 \%)$ & $13(54.17 \%)$ & \\
\hline Valvular & $3(4.41 \%)$ & $2(8.33 \%)$ & \\
\hline Hypertrophic cardiomyopathy & $0(0.00 \%)$ & $2(8.33 \%)$ & \\
\hline Non-compaction & $2(2.94 \%)$ & $0(0.00 \%)$ & \\
\hline Post chemotherapy & $2(2.94 \%)$ & $1(4.17 \%)$ & \\
\hline Post-myocarditis - Inflammatory & $4(5.88 \%)$ & $0(0.00 \%)$ & \\
\hline Ethyl / Toxic & $4(5.88 \%)$ & $2(8.33 \%)$ & \\
\hline Idiopathic dilated cardiomyopathy & $19(27.94 \%)$ & $3(12.50 \%)$ & \\
\hline Peri-partum & $1(1.47 \%)$ & $0(0.00 \%)$ & $1(4.17 \%)$ \\
\hline Congenital & $3(4.41 \%)$ & $23(95.8 \%)$ & \\
\hline INTERMACS scale & & $1(4.2 \%)$ & \\
\hline I-III & $60(88.2 \%)$ & & \\
\hline IV & $8(11.8 \%)$ & & \\
\hline Pre-implant treatment & & & \\
\hline
\end{tabular}

Values are represented as number (\%), or mean \pm SD - median [interquartile range]

P-values: $\mathrm{Chi}^{2}$ test or Mann-Whitney test $\mathrm{p}$-values

INTERMACS, interagency registry for mechanically assisted circulatory support; BMI, body mass index, ECMO, extracorporeal membrane oxygenation; IABP, intra-aortic balloon pump; AST, aspartate transaminase; ALT, alanine transaminase; INR, international normalized ratio; LVEF, left ventricular ejection fraction; TAPSE, tricuspid annular plane systolic excursion; HR, heart rate; SBP, systolic blood pressure; DBP, diastolic blood pressure; MAP, mean atrial pressure; RAP, right atrial pressure; PAPs, pulmonary artery systolic pressure; PAPd, pulmonary artery diastolic pressure; PAPm, pulmonary artery mean pressure; PVR, pulmonary vascular resistance; PCWP, pulmonary capillary wedge pressure; TPG, transpulmonary gradient; DPG, diastolic pulmonary gradient; $\mathrm{CO}$, cardiac output; $\mathrm{Cl}$, cardiac index; SV, stroke volume; SI, stroke index; PAPi, pulmonary artery pulsatility index; RVSWI, right ventricular stroke work index; Cpa, compliance pulmonary artery. 


\begin{tabular}{|c|c|c|c|}
\hline Variables & $\begin{array}{l}\text { No severe RV failure } \\
(n=68)\end{array}$ & $\begin{array}{l}\text { Severe RV failure } \\
(n=24)\end{array}$ & P-value \\
\hline Amiodarone $(\mathrm{Y} / \mathrm{N})$ & $\begin{array}{l}22 / 45(32.8 \% / \\
67.2 \%)\end{array}$ & 12 / $11(52.2 \%$ / 47.8\%) & 0.098 \\
\hline ACE inhibitors $(\mathrm{Y} / \mathrm{N})$ & $\begin{array}{l}53 / 12(81.5 \% / \\
18.5 \%)\end{array}$ & 16 / 7 (69.6\% / 30.4\%) & 0.23 \\
\hline Beta-blockers $(\mathrm{Y} / \mathrm{N})$ & $\begin{array}{l}55 / 12(82.1 \% / \\
17.9 \%)\end{array}$ & 20 / $2(90.9 \%$ / 9.1\%) & 0.32 \\
\hline Aldactone $(\mathrm{Y} / \mathrm{N})$ & $\begin{array}{l}41 / 25(62.1 \% / \\
37.9 \%)\end{array}$ & 17 / 6 (73.9\% / 26.1\%) & 0.30 \\
\hline $\begin{array}{l}\text { Angiotensin Receptor Inhibitors } \\
(\mathrm{Y} / \mathrm{N})\end{array}$ & $3 / 64(4.5 \%$ / 95.5\%) & 2 / 20 (9.1\% / 90.9\%) & 0.41 \\
\hline Loop diuretics $(\mathrm{Y} / \mathrm{N})$ & 58 / 9 (86.6\% / 13.4\%) & $21 / 2(91.3 \% / 8.7 \%)$ & 0.55 \\
\hline Anticoagulants & & & 0.13 \\
\hline No & $36(57.1 \%)$ & $7(31.8 \%)$ & \\
\hline Vitamin $\mathrm{K}$ antagonist & $22(34.9 \%)$ & $10(45.5 \%)$ & \\
\hline NOAC & $3(4.8 \%)$ & $3(13.6 \%)$ & \\
\hline Heparine & $2(3.2 \%)$ & $2(9.1 \%)$ & \\
\hline Any Anticoagulant $(\mathrm{Y} / \mathrm{N})$ & $\begin{array}{l}27 / 36(42.9 \% / \\
57.1 \%)\end{array}$ & 15 / 7 (68.2\% / 31.8\%) & 0.04 \\
\hline $\begin{array}{l}\text { Implantable cardioverter- } \\
\text { defibrillator }(\mathrm{Y} / \mathrm{N})\end{array}$ & $\begin{array}{l}21 / 47(30.9 \% / \\
69.1 \%)\end{array}$ & $8 / 15$ (34.8\% / 65.2\%) & 0.73 \\
\hline \multicolumn{4}{|l|}{ Organ Failure support } \\
\hline Number inotropes before LVAD & $1.51 \pm 0.63-2[1-2]$ & $2.25 \pm 0.67-2[2-3]$ & 0.00003 \\
\hline $\begin{array}{l}\text { Renal replacement therapy before } \\
\text { LVAD (Yes/No) }\end{array}$ & $4 / 64(5.9 \%$ / 94.1\%) & $5 / 19(20.8 \% / 79.2 \%)$ & 0.034 \\
\hline \multicolumn{4}{|c|}{ Values are represented as number (\%), or mean $\pm \mathrm{SD}-$ median [interquartile range] } \\
\hline \multicolumn{4}{|c|}{ P-values: $\mathrm{Chi}^{2}$ test or Mann-Whitney test $\mathrm{p}$-values } \\
\hline \multicolumn{4}{|c|}{$\begin{array}{l}\text { INTERMACS, interagency registry for mechanically assisted circulatory support; BMI, body mass } \\
\text { index, ECMO, extracorporeal membrane oxygenation; IABP, intra-aortic balloon pump; AST, aspartate } \\
\text { transaminase; ALT, alanine transaminase; INR, international normalized ratio; LVEF, left ventricular } \\
\text { ejection fraction; TAPSE, tricuspid annular plane systolic excursion; HR, heart rate; SBP, systolic blood } \\
\text { pressure; DBP, diastolic blood pressure; MAP, mean atrial pressure; RAP, right atrial pressure; PAPs, } \\
\text { pulmonary artery systolic pressure; PAPd, pulmonary artery diastolic pressure; PAPm, pulmonary } \\
\text { artery mean pressure; PVR, pulmonary vascular resistance; PCWP, pulmonary capillary wedge } \\
\text { pressure; TPG, transpulmonary gradient; DPG, diastolic pulmonary gradient; CO, cardiac output; Cl, } \\
\text { cardiac index; SV, stroke volume; SI, stroke index; PAPi, pulmonary artery pulsatility index; RVSWI, right } \\
\text { ventricular stroke work index; Cpa, compliance pulmonary artery. }\end{array}$} \\
\hline
\end{tabular}




\begin{tabular}{|c|c|c|c|}
\hline Variables & $\begin{array}{l}\text { No severe RV failure } \\
(n=68)\end{array}$ & $\begin{array}{l}\text { Severe RV failure } \\
(n=24)\end{array}$ & P-value \\
\hline $\begin{array}{l}\text { Mechanical ventilation before } \\
\text { LVAD (Yes/No) }\end{array}$ & $\begin{array}{l}15 / 53(22.1 \% / \\
77.9 \%)\end{array}$ & $13 / 11(54.2 \%$ / 45.8\%) & 0.0033 \\
\hline $\operatorname{ECMO}(\mathrm{Y} / \mathrm{N})$ & $\begin{array}{l}13 / 55(19.1 \% / \\
80.9 \%)\end{array}$ & 10 / 14 (41.7\% / 58.3\%) & 0.028 \\
\hline IABP $(\mathrm{Y} / \mathrm{N})$ & $4 / 64(5.9 \%$ / 94.1\%) & 2/22 (8.3\% / 91.7\%) & 0.67 \\
\hline \multicolumn{4}{|l|}{ Laboratory values } \\
\hline creatinine & $1.2 \pm 0.6-1.2[1-1.4]$ & $1.5 \pm 0.6-1.4[1.1-1.8]$ & 0.028 \\
\hline bilirubine & $1 \pm 0.6-0.9[0.5-1.4]$ & $1.6 \pm 1.1-1.3[0.8-2.1]$ & 0.034 \\
\hline hemoglobin & $11.2 \pm 2.1-11[9.3-13]$ & $9.9 \pm 1.9-9.1[8.6-11.5]$ & 0.018 \\
\hline AST & $\begin{array}{l}54.1 \pm 126-22[17- \\
39.5]\end{array}$ & $\begin{array}{l}67.6 \pm 77.4-35.5[22.3- \\
67.5]\end{array}$ & 0.019 \\
\hline ALT & $85.2 \pm 251-30[17-60]$ & $\begin{array}{l}72.3 \pm 104.6-35.5[21- \\
83]\end{array}$ & 0.39 \\
\hline INR & $1.3 \pm 0.4-1.2[1.1-1.3]$ & $1.3 \pm 0.2-1.3[1.2-1.4]$ & 0.059 \\
\hline White Blood Cell count & $7.7 \pm 3.1-7[6-9]$ & $8.4 \pm 3.9-7.3[5.7-11]$ & 0.71 \\
\hline NTproBNP & $\begin{array}{l}8006 \pm 9169- \\
4379[2470-8831]\end{array}$ & $\begin{array}{l}10554 \pm 6483-8900 \\
{[5994-12950]}\end{array}$ & 0.011 \\
\hline \multicolumn{4}{|l|}{ Hemodynamics } \\
\hline$H R$ & $\begin{array}{l}88.2 \pm 20.1-89 \text { [70.3- } \\
101.5]\end{array}$ & $\begin{array}{l}85.2 \pm 16.8-179.5 \\
{[72.5-98.8]}\end{array}$ & 0.62 \\
\hline SBP & $\begin{array}{l}102.4 \pm 14.6-101 \\
{[93.8-110]}\end{array}$ & $\begin{array}{l}105.5 \pm 15.9-104 \text { [94- } \\
115]\end{array}$ & 0.51 \\
\hline DBP & $\begin{array}{l}66.2 \pm 10.8-65[60- \\
73]\end{array}$ & $65.7 \pm 10.2-67[58-74]$ & 0.88 \\
\hline \multicolumn{4}{|c|}{ Values are represented as number (\%), or mean \pm SD - median [interquartile range] } \\
\hline \multicolumn{4}{|c|}{ P-values: Chi ${ }^{2}$ test or Mann-Whitney test $p$-values } \\
\hline \multicolumn{4}{|c|}{$\begin{array}{l}\text { INTERMACS, interagency registry for mechanically assisted circulatory support; BMI, body mass } \\
\text { index, ECMO, extracorporeal membrane oxygenation; IABP, intra-aortic balloon pump; AST, aspartate } \\
\text { transaminase; ALT, alanine transaminase; INR, international normalized ratio; LVEF, left ventricular } \\
\text { ejection fraction; TAPSE, tricuspid annular plane systolic excursion; HR, heart rate; SBP, systolic blood } \\
\text { pressure; DBP, diastolic blood pressure; MAP, mean atrial pressure; RAP, right atrial pressure; PAPs, } \\
\text { pulmonary artery systolic pressure; PAPd, pulmonary artery diastolic pressure; PAPm, pulmonary } \\
\text { artery mean pressure; PVR, pulmonary vascular resistance; PCWP, pulmonary capillary wedge } \\
\text { pressure; TPG, transpulmonary gradient; DPG, diastolic pulmonary gradient; CO, cardiac output; Cl, } \\
\text { cardiac index; SV, stroke volume; SI, stroke index; PAPi, pulmonary artery pulsatility index; RVSWI, right } \\
\text { ventricular stroke work index; Cpa, compliance pulmonary artery. }\end{array}$} \\
\hline
\end{tabular}




\begin{tabular}{|llll|}
\hline Variables & $\begin{array}{l}\text { No severe RV failure } \\
(\mathbf{n}=68)\end{array}$ & $\begin{array}{l}\text { Severe RV failure } \\
(\mathbf{n}=\mathbf{2 4})\end{array}$ & P-value \\
\hline MAP & $78 \pm 10.6-77[72-85]$ & $78.2 \pm 8.1-77[71-84]$ & 1.00 \\
\hline RAP & $9.3 \pm 4.2-9[6-12]$ & $13.8 \pm 5.6-14[9-19]$ & 0.0009 \\
\hline PAPs & $49.8 \pm 13.3-49.5[40-$ & $52.9 \pm 20.4-56[36.5-$ & 0.42 \\
\hline PAPd & $59.8]$ & $60.8]$ & \\
\hline PAPm & $26.4 \pm 7.5-26[21-$ & $28.9 \pm 10.9-28[20.5-$ & 0.38 \\
\hline PCWP & $32.3]$ & $36.8]$ & 0.48 \\
\hline TPG & $34.6 \pm 8.8-34.5[28-$ & $37.4 \pm 13.3-36[26.8-$ & \\
\hline DPG & $39.8]$ & $45]$ & 0.93 \\
\hline CO & $23.1 \pm 6.7-23[19-28]$ & $22.9 \pm 8.4-24[15.3-$ & 0.32 \\
\hline Cl & $11.7 \pm 5.3-11[8-15]$ & $14.5 \pm 8.3-12[10-19.8]$ & 0.25 \\
\hline SV & $3.7 \pm 4.7-3[1.3-5]$ & $6 \pm 6.4-4[2-9]$ & 0.043 \\
\hline SI & $3.9 \pm 1.2-3.8[2.9-4.6]$ & $3.3 \pm 1-3[2.6-3.8]$ & 0.014 \\
\hline PVR & $2.1 \pm 0.6-2.1[1.7-2.4]$ & $1.8 \pm 0.4-1.7[1.5-2.2]$ & 0.0028 \\
\hline RAP/PCWP & $46 \pm 18-43[33-57.5]$ & $40 \pm 13.8-38[32.3-49]$ & 0.18 \\
\hline PAPi & $24.8 \pm 8.2-25[18.5-$ & $21.4 \pm 5.7-21.8[16.6-$ & 0.062 \\
\hline RVSWI & $28.3]$ & $24]$ & 0.15 \\
\hline Cpa & $3.2 \pm 1.5-3[2.2-4]$ & $4.6 \pm 3.2-4.1[2.1-5.2]$ & 0.088 \\
\hline & $0.4 \pm 0.1-0.4[0.3-0.5]$ & $0.7 \pm 0.3-0.6[0.5-0.8]$ & $\mathbf{0 . 0 0 0 0 1}$ \\
\hline $3.3 \pm 2.6-2.4[1.8-4.2]$ & $2 \pm 1.5-1.7[1-2.3]$ & $\mathbf{0}$ \\
\hline
\end{tabular}

Values are represented as number (\%), or mean \pm SD - median [interquartile range]

P-values: $\mathrm{Chi}^{2}$ test or Mann-Whitney test $\mathrm{p}$-values

INTERMACS, interagency registry for mechanically assisted circulatory support; BMI, body mass index, ECMO, extracorporeal membrane oxygenation; IABP, intra-aortic balloon pump; AST, aspartate transaminase; ALT, alanine transaminase; INR, international normalized ratio; LVEF, left ventricular ejection fraction; TAPSE, tricuspid annular plane systolic excursion; HR, heart rate; SBP, systolic blood pressure; DBP, diastolic blood pressure; MAP, mean atrial pressure; RAP, right atrial pressure; PAPs, pulmonary artery systolic pressure; PAPd, pulmonary artery diastolic pressure; PAPm, pulmonary artery mean pressure; PVR, pulmonary vascular resistance; PCWP, pulmonary capillary wedge pressure; TPG, transpulmonary gradient; DPG, diastolic pulmonary gradient; $\mathrm{CO}$, cardiac output; $\mathrm{Cl}$, cardiac index; SV, stroke volume; SI, stroke index; PAPi, pulmonary artery pulsatility index; RVSWI, right ventricular stroke work index; Cpa, compliance pulmonary artery. 


\begin{tabular}{|c|c|c|c|}
\hline Variables & $\begin{array}{l}\text { No severe RV failure } \\
(n=68)\end{array}$ & $\begin{array}{l}\text { Severe RV failure } \\
(n=24)\end{array}$ & P-value \\
\hline $\mathrm{SvO} 2$ & $\begin{array}{l}62.1 \pm 11-62[54.8- \\
69]\end{array}$ & $\begin{array}{l}51.9 \pm 7.4-53[45.3- \\
59.5]\end{array}$ & 0.00037 \\
\hline SatO2 & $\begin{array}{l}96.6 \pm 3.7-97.5[94.5- \\
99]\end{array}$ & $97.5 \pm 2-97$ [96-99] & 0.76 \\
\hline \multicolumn{4}{|l|}{ Echocardiographic } \\
\hline LVEF & $\begin{array}{l}22.1 \pm 6.1-22.5[20- \\
25]\end{array}$ & $25 \pm 7.9-25[20-30]$ & 0.14 \\
\hline Right Ventricular enlargement & & & 0.027 \\
\hline No & $28(50.9 \%)$ & $7(38.9 \%)$ & \\
\hline Mild & $11(20.0 \%)$ & $1(5.6 \%)$ & \\
\hline Moderate & $16(29.1 \%)$ & $8(44.4 \%)$ & \\
\hline Severe & $0(0.0 \%)$ & $2(11.1 \%)$ & \\
\hline Right Ventricular dysfunction & & & 0.10 \\
\hline No & $15(23.4 \%)$ & $4(17.4 \%)$ & \\
\hline Mild / Moderate & $38(59.4 \%)$ & $10(43.5 \%)$ & \\
\hline Severe & $11(17.2 \%)$ & $8(39.1 \%)$ & \\
\hline Tricuspid valve regurgitation & & & 0.49 \\
\hline No & $4(7.4 \%)$ & $3(15.0 \%)$ & \\
\hline Trivial / Mild & $22(40.7 \%)$ & $5(25.0 \%)$ & \\
\hline Moderate & $21(38.9 \%)$ & $10(50.0 \%)$ & \\
\hline Severe & $7(10.0 \%)$ & $2(10.0 \%)$ & \\
\hline
\end{tabular}

Values are represented as number (\%), or mean \pm SD - median [interquartile range]

P-values: $\mathrm{Chi}^{2}$ test or Mann-Whitney test $\mathrm{p}$-values

INTERMACS, interagency registry for mechanically assisted circulatory support; BMI, body mass index, ECMO, extracorporeal membrane oxygenation; IABP, intra-aortic balloon pump; AST, aspartate transaminase; ALT, alanine transaminase; INR, international normalized ratio; LVEF, left ventricular ejection fraction; TAPSE, tricuspid annular plane systolic excursion; HR, heart rate; SBP, systolic blood pressure; DBP, diastolic blood pressure; MAP, mean atrial pressure; RAP, right atrial pressure; PAPs, pulmonary artery systolic pressure; PAPd, pulmonary artery diastolic pressure; PAPm, pulmonary artery mean pressure; PVR, pulmonary vascular resistance; PCWP, pulmonary capillary wedge pressure; TPG, transpulmonary gradient; DPG, diastolic pulmonary gradient; $\mathrm{CO}$, cardiac output; $\mathrm{Cl}$, cardiac index; SV, stroke volume; SI, stroke index; PAPi, pulmonary artery pulsatility index; RVSWI, right ventricular stroke work index; Cpa, compliance pulmonary artery. 


\begin{tabular}{|c|c|c|c|}
\hline Variables & $\begin{array}{l}\text { No severe RV failure } \\
(n=68)\end{array}$ & $\begin{array}{l}\text { Severe RV failure } \\
(n=24)\end{array}$ & P-value \\
\hline \multicolumn{3}{|l|}{ Mitral valve regurgitation } & 0.19 \\
\hline No & $3(4.5 \%)$ & \multicolumn{2}{|l|}{$2(9.1 \%)$} \\
\hline Trivial / Mild & $15(22.4 \%)$ & \multicolumn{2}{|l|}{$6(27.3 \%)$} \\
\hline Moderate & $30(44.8 \%)$ & \multicolumn{2}{|l|}{$13(59.1 \%)$} \\
\hline Moderate / Severe & $11(16.4 \%)$ & \multicolumn{2}{|l|}{$0(0.0 \%)$} \\
\hline Severe & $8(11.9 \%)$ & \multicolumn{2}{|l|}{$1(4.6 \%)$} \\
\hline TAPSE & $\begin{array}{l}15.2 \pm 4.4-14.5[13- \\
18]\end{array}$ & $\begin{array}{l}13.3 \pm 3.8-13.5[10.8- \\
15.3]\end{array}$ & 0.13 \\
\hline TAPSE/PAPs & $\begin{array}{l}0.33 \pm 0.16-0.31 \\
{[0.24-0.40]}\end{array}$ & $\begin{array}{l}0.32 \pm 0.19-0.33[0.22- \\
0.39]\end{array}$ & 0.71 \\
\hline $\mathrm{RV} / \mathrm{LV}$ diameters & $\begin{array}{l}0.58 \pm 0.12-0.56 \\
{[0.49-0.67]}\end{array}$ & $\begin{array}{l}0.66 \pm 0.13-0.70[0.54- \\
0.74]\end{array}$ & 0.04 \\
\hline $\begin{array}{l}\text { Inferior vena cava dilatation } \\
\text { (Yes/No) }\end{array}$ & $\begin{array}{l}27 / 26(50.9 \% / \\
40.1 \%)\end{array}$ & 17 / 3 (85.0\% / 15.0\%) & 0.008 \\
\hline \multicolumn{4}{|c|}{ Values are represented as number (\%), or mean \pm SD - median [interquartile range] } \\
\hline \multicolumn{4}{|c|}{ P-values: Chi ${ }^{2}$ test or Mann-Whitney test $p$-values } \\
\hline \multicolumn{4}{|c|}{$\begin{array}{l}\text { INTERMACS, interagency registry for mechanically assisted circulatory support; BMI, body mass } \\
\text { index, ECMO, extracorporeal membrane oxygenation; IABP, intra-aortic balloon pump; AST, aspartate } \\
\text { transaminase; ALT, alanine transaminase; INR, international normalized ratio; LVEF, left ventricular } \\
\text { ejection fraction; TAPSE, tricuspid annular plane systolic excursion; HR, heart rate; SBP, systolic blood } \\
\text { pressure; DBP, diastolic blood pressure; MAP, mean atrial pressure; RAP, right atrial pressure; PAPs, } \\
\text { pulmonary artery systolic pressure; PAPd, pulmonary artery diastolic pressure; PAPm, pulmonary } \\
\text { artery mean pressure; PVR, pulmonary vascular resistance; PCWP, pulmonary capillary wedge } \\
\text { pressure; TPG, transpulmonary gradient; DPG, diastolic pulmonary gradient; CO, cardiac output; Cl, } \\
\text { cardiac index; SV, stroke volume; SI, stroke index; PAPi, pulmonary artery pulsatility index; RVSWI, right } \\
\text { ventricular stroke work index; Cpa, compliance pulmonary artery. }\end{array}$} \\
\hline
\end{tabular}

Median age was 53 years-old (interquartile range 36-60), 71 (77\%) patients were white, and 68 (74\%) were males. Aetiology of HF was primarily ischemic in 37 patients (40\%) followed by dilated cardiomyopathy in 22 patients (24\%) and others causes in 33 patients (36\%).

RHF was mostly observed in patients with a higher-acuity INTERMACS level, with a greater preoperative inotropes' requirement, as well as with a greater need of renal replacement therapy, mechanical ventilation and temporary MCS. 
They had significantly higher preoperative creatinine, bilirubin and aspartate transaminase, and lower haemoglobin. The level of N-terminal pro-brain natriuretic peptide (NTproBNP) was as well significantly higher in the group presenting a severe RHF.

For right heart catheterization parameters, a higher RAP, higher RA/PWCP ratio and lower PAPi were predictive of RHF. A significantly lower cardiac index and SvO2 were also present in the group of severe RHF. A RV enlargement with a greater ratio of RV/LV diameters and inferior vena cava dilatation observed by echocardiographic analysis, were found in the RHF group.

Patients with severe RHF had higher early post-operatory mortality and more frequent complications (Table 3).

Table 3

Patient Outcomes and adverse events

\begin{tabular}{|c|c|c|c|c|c|}
\hline & \multicolumn{2}{|c|}{ No severe RV failure } & \multicolumn{3}{|c|}{ Severe RV failure } \\
\hline & Number & Percent & Number & Percent & $\begin{array}{l}\text { Chi- } \\
\text { square } \\
\text { test }\end{array}$ \\
\hline & & & & & $\mathrm{p}$-value \\
\hline Discharge alive $(\mathrm{Y} / \mathrm{N})$ & $61 / 7$ & $89.7 \%$ / 10.3\% & $13 / 11$ & $54.2 \% / 45.8 \%$ & 0.0002 \\
\hline Early infection (Y/N/UNK) & $\begin{array}{l}39 / 28 \\
/ 1\end{array}$ & $\begin{array}{l}57.3 \% / 41.2 \% \\
/ 1.5 \%\end{array}$ & $\begin{array}{l}19 / 4 \\
/ 1\end{array}$ & $\begin{array}{l}79.2 \% / 16.7 \% \\
/ 4.2 \%\end{array}$ & 0.0823 \\
\hline Major bleeding $(\mathrm{Y} / \mathrm{N})$ & 20 / 48 & $29.4 \% / 70.6 \%$ & $8 / 16$ & $33.3 \%$ / 66.7\% & 0.719 \\
\hline $\begin{array}{l}\text { Pericardial fluid collection } \\
\text { (Y/N/UNK) }\end{array}$ & $\begin{array}{l}15 / 51 \\
/ 2\end{array}$ & $\begin{array}{l}22.1 \% / 75 \% / \\
2.9 \%\end{array}$ & $\begin{array}{l}1 / 23 / \\
0\end{array}$ & $\begin{array}{l}4.2 \% / 19.3 \% / \\
0 \%\end{array}$ & 0.0851 \\
\hline $\begin{array}{l}\text { Neurological dysfunction } \\
\text { (Y/N/UNK) }\end{array}$ & $\begin{array}{l}11 / 84 \\
/ 3\end{array}$ & $\begin{array}{l}16.2 \% / 79.4 \% \\
/ 4.4 \%\end{array}$ & $\begin{array}{l}8 / 12 / \\
4\end{array}$ & $\begin{array}{l}33.3 \% / 50 \% / \\
16.7 \%\end{array}$ & 0.0168 \\
\hline $\begin{array}{l}\text { Cardiac arrhythmias } \\
\text { (Y/N/UNK) }\end{array}$ & $\begin{array}{l}36 / 26 \\
/ 6\end{array}$ & $\begin{array}{l}52.9 \% / 38.2 \% \\
/ 8.8 \%\end{array}$ & $\begin{array}{l}17 / 4 \\
/ 3\end{array}$ & $\begin{array}{l}70.8 \% / 16.7 \% \\
/ 12.5 \%\end{array}$ & 0.152 \\
\hline $\begin{array}{l}\text { Myocardial infarction } \\
\text { (Y/N/UNK) }\end{array}$ & $\begin{array}{l}0 / 66 \\
/ 2\end{array}$ & $\begin{array}{l}0 \% / 97.1 \% / \\
2.9 \%\end{array}$ & $\begin{array}{l}0 / 22 / \\
2\end{array}$ & $\begin{array}{l}0 \% / 91.7 \% / \\
8.3 \%\end{array}$ & 0.265 \\
\hline $\begin{array}{l}\text { Respiratory failure } \\
\text { (Y/N/UNK) }\end{array}$ & $\begin{array}{l}18 / 46 \\
/ 4\end{array}$ & $\begin{array}{l}26.5 \% / 67.6 \% \\
/ 5.9 \%\end{array}$ & $\begin{array}{l}15 / 7 / \\
2\end{array}$ & $\begin{array}{l}62.5 \% / 19.2 \% \\
/ 8.3 \%\end{array}$ & 0.0038 \\
\hline $\begin{array}{l}\text { Acute kidney injury } \\
\text { (Y/N/UNK) }\end{array}$ & $\begin{array}{l}37 / 30 \\
/ 1\end{array}$ & $\begin{array}{l}34.4 \% / 44.1 \% \\
/ 1.5 \%\end{array}$ & $\begin{array}{l}22 / 1 / \\
1\end{array}$ & $\begin{array}{l}91.7 \% / 4.2 \% / \\
4.1 \%\end{array}$ & 0.0016 \\
\hline
\end{tabular}

In-hospital death occurred in 18 patients (20\%) after LVAD implantation, with mortality rates of $46 \%$ in the RHF group and $10 \%$ in patients without RHF $(p=0.0002)$. Three of the thirteen patients who survived in the RHF group needed a high urgent HT between 12 and 40 days after LVAD implantation. 
Incidences of respiratory failure ( $62 \%$ vs $26 \%, p=0.003)$, acute kidney injury ( $92 \%$ vs $34 \%, p=0.001)$ and neurological dysfunction ( $33 \%$ vs $16 \%, p=0.01$ ) were significantly higher in patients with RHF compared to patients without.

\section{Right Heart Failure and Risk Models}

The variables included in the univariate logistic regression model are presented in Supplemental Table 1.

The four variables for severe RHF selected for the best reduced logistic model in our population are presented in table 4: the high INTERMACS level (class 1 to 3$)(p=0.19)$, the number of inotropes used ( $p$ $=0.006)$, the ratio of right atrial pressure/pulmonary capillary wedge pressure (RAP/PWCP) at the right heart catheterization $(p=0.01)$ and the ratio of right ventricle/left ventricle $(R V / L V)$ diameters in four chamber apical echocardiogram view (0.39).

Table 4

Results of Multivariable Logistic Regression Analysis

\begin{tabular}{|lllll|}
\hline Severe ventricular failure & & & & \\
\hline Independent Variable & Odds ratio & Lower 95\% Cl & Upper 95\% Cl & Wald p-value \\
\hline Constant & 0.00001 & 0.00000 & 0.00515 & $\mathbf{0 . 0 0 0 2 9}$ \\
\hline Number of inotropes & 6.9 & 1.7 & 27.7 & $\mathbf{0 . 0 0 6 2 7}$ \\
\hline RAP/PCWP & 487.9 & 4.3 & $10000+$ & $\mathbf{0 . 0 1}$ \\
\hline RV / LV diameters & 18.6 & 0.02 & $10000+$ & 0.39 \\
\hline INTERMACS scale = 1 to 3 & 5.6 & 0.4 & 77.5 & 0.19 \\
Cl = confidence interval & & & & \\
\hline RV = right ventricle & & & & \\
\hline LV = left ventricle & & & \\
\hline RAP = right atrial pressure & & & \\
\hline PCWP = pulmonary capillary wedge pressure & & \\
\hline
\end{tabular}

This model demonstrated a significant improvement in prediction of RHF. As shown in Fig. 1, the area under ROC curve for severe RHF was 0.91 (Cl: 0.76-0.96); in the jitter plot in Fig. 2, patients with severe $\mathrm{RHF}$ are represented on the right side of the $\mathrm{x}$-axis of probability.

In the comparison of the three scores of RHF, the EUROMACS-RHF risk score (C $=0.82-95 \% \mathrm{Cl}$ : $0.68-$ $0.90)$ performed best in predicting RHF compared to the Michigan RVF score $(C=0.69-95 \% \mathrm{Cl}$ : $0.54-$ 
0.80). However, The CRITT score performed best in predicting the need of RVAD placement $(C=0.86-$ 95\% Cl: 0.72-0.93) (Fig. 3).

\section{Discussion}

In this contemporary cohort of patients undergoing primary implantation of continuous-flow LVADs, we found that the EUROMACS-RHF risk score performed best in predicting right heart failure. Furthermore, we created a model with four preoperative metrics, which demonstrated good predictive ability in our population.

RHF is an important and frequent complication in the early postoperative period after LVAD implantation. In prior studies, rates of post-LVAD RHF have ranged from 4-50\% ${ }^{10-15}$. This wide range of reported RHF incidence is due to the heterogeneities in RHF definitions. Our definition was in line with the INTERMACS definition of severe RHF ${ }^{16}$ and was limited to the immediate postoperative period after LVAD implantation.

In our study, the incidence of RHF was $26 \%$, similar to the recently published EUROMACS study ${ }^{23}$.

Furthermore, patients who develop RHF have also a greater risk to develop concomitant complications even death ${ }^{13-24}$.

In our analysis, RHF was associated with a greater post-operatory mortality rate, as well as with additional comorbidities such as cerebrovascular accidents, respiratory failure, acute kidney injury and multiorgan failure.

If we consider two different groups of patients regarding post- LVAD RHF and its complications, the challenge for the medical team is to determine which predictive parameters they differ from.

Numerous pre-operative risk scores have been developed to quantify the risk of RHF in LVAD candidates $20-25$. With the exception of the EUROMACS RHF risk score, most predictors scores were typically developed in small single-center studies, used various definitions of RHF, the heterogeneous nature of LVADs leading to inconsistent predictors and no single model dependably forecasting RHF. Moreover, there is a paucity of external validation studies on these risk prediction models ${ }^{24}$.

The well-known and most used risk scores include the Michigan RVF score ${ }^{21}$, the Heartmate II bridge-totransplantation RVF analysis ${ }^{13}$, the Utah RVF risk score ${ }^{20}$, the Pittsburgh decision tree ${ }^{22}$, the CRITT score ${ }^{14}$ and the EUROMACS RHF-risk score ${ }^{23}$.

Results from these studies have reported varying predictive ability of RHF, but with no ideal C-statistics, mostly ranging from 0.6 to 0.7 . 
Peter et al. ${ }^{24}$, have recently compared the performance of some of these risk scores, finding the Michigan score as the only risk model to demonstrate significant discrimination for RHF, even if modest $(C=0.74)$, compared with newer risk scores (Utah, Pitt, EUROMACS-RHF).

In a recent meta-analysis, Bellavia et al. ${ }^{26}$ evaluated observational studies of risk factors associated with RHF after LVAD implantation. Variables found with the highest effect size in predicting RHF were: the need for mechanical ventilation and continuous renal replacement therapy, international normalized ratio and NT-proBNP, RV stroke work index and central venous pressure, pre-implant moderate to severe RV dysfunction and greater RV/LV diameter ratio.

In our study, we performed a comparative analysis between the risk scores mentioned above. We excluded the Utah risk score as it retains the presence of DT which was not used in our population, and the Pittsburgh score due the lacking of several parameters. Finally, we thus compared the Michigan score, the CRITT score and the EUROMACS-RHF score.

In our comparative analysis of these three scores, the EUROMACS-RHF risk score performed the best in predicting RHF.

EUROMACS-RHF ${ }^{23}$ is a simple risk score comprising a range of variables including cardiopulmonary hemodynamic and echocardiographic metrics, patient characteristics, and preoperative medical management. It was derived from and validated by more than 2000 adults who underwent continuousflow LVAD implantation across the European Union in the largest EU Registry of mechanical circulatory support devices.

However, one of the limitations of this score is the semiqualitative assessment of RV function on echocardiography evaluated by the visual aspect of RV function.

In our analysis, the most significant predictors of RHF, were the INTERMACS level, the use of a high number of inotropes, the RAP/PWCP ratio and the RV/LV diameters ratio.

The difference obtained with our analysis compared to EUROMACS-RHF was a quantitative RV assessment in echocardiography obtained by the ratio of the RV and LV diameters.

The present study validates the most used and recent RHF score in a continuous flow LVAD population and expands the comparative analysis to include newer predictive models and metrics.

We highlight the severity of the population who develop RHF by the high INTERMACS level and the need for multiples inotropes, with a focus on the right ventricle itself by the dilatation and the elevation of right pressures.

Judicious patient selection is vital to preventing RHF in patients undergoing LVAD implantation. 
A patient with a high-risk RHF score may require perioperative optimization of RV support ${ }^{29}$ via reduction of preload ${ }^{30,31}$, afterload ${ }^{32}$, and RV contractility support ${ }^{33}$, or early RV mechanical support or biventricular assist device ${ }^{34,35}$.

In fact, despite aggressive risk stratification and medical management, some patients still develop RHF requiring RVAD support. The need for an RVAD is associated with more severe outcomes ${ }^{34}$ and an elective RVAD correlates with better long-term survival than an emergency implantation ${ }^{36}$, whilst also improving survival to transplant compared with delayed RVAD insertion ${ }^{37}$. The use of those predictive RHF score help medical team to better prepare high risk patients and to be more quickly reactive to complications.

\section{Limitations}

This study is a single-center retrospective study. The sample size is fairly modest and the analyzed population was further reduced by missing hemodynamic variables in some of the patients.

In addition, this study was unable to compare the UTAH and the Pittsburgh model. Innovative parameters to evaluate the RV as the speckle tracking echocardiography (strain), the 3D-RV echocardiography, or the cardiac MRI were not studied to improve the evaluation of RV dysfunction.

\section{Conclusion}

Amongst the available risk scores, the EUROMACS performs best to predict the occurrence of RHF after LVAD implantation. Based on 4 simple metrics, our model's performance compares well to the EUROMACS score, adding a more objective quantitative evaluation of pre-operatory RV function.

\section{List Of Abbreviations}

AST, aspartate transaminase

$\mathrm{ALT}$, alanine transaminase

CO, cardiac output

$\mathrm{Cl}$, cardiac index

ECMO, extracorporeal membrane oxygenation

$\mathrm{HR}$, heart rate

IABP, intra-aortic balloon pump

INR, international normalized ratio 
INTERMACS, interagency registry for mechanically assisted circulatory support

iNO, inhaled nitric oxide

LVEF, left ventricular ejection fraction

LVAD left ventricular assist device

TAPSE, tricuspid annular plane systolic excursion

PAPs, pulmonary artery systolic pressure

PAPd, pulmonary artery diastolic pressure

PAPm, pulmonary artery mean pressure

PVR, pulmonary vascular resistance

PCWP, pulmonary capillary wedge pressure

PAPi, pulmonary artery pulsatility index

RAP, right atrial pressure

RVAD right ventricular assist device

RVSWI, right ventricular stroke work index

TAPSE, tricuspid annular plane systolic excursion

\section{Declarations}

\section{Ethics approval and consent to participate}

Ethics commette approval of retrospective study non interventional (database analysis)

Ethics commette Erasme Hospital

References: P2019/670/NA

\section{Consent for publication}

Collection of existing data in the medical records of the target persons having not expressed opposition to the use of their medical records for the purpose for research purposes.

Exemption from the principle of patient consent approved from ethics committee. Justification: Obtaining consent unrealistic given the number of files involved and the likelihood of having many patients who are 
no longer reachable. Risk of calling a family to process the data of a deceased person.

\section{Availability of data and materials}

The datasets used and analysed during the current study are available from the corresponding author on reasonable request.

\section{Competing interests}

The authors declare that they have no competing interests.

\section{Funding}

No funding.

\section{Authors' contributions}

Conceptualization: Federica Valente, Constantin Stefanidis, Jean-Luc Vachiéry, Céline Dewachter, Ana Roussoulières

Data curation: Federica Valente, Edgard Engelman

Formal analysis: Edgard Engelman

Investigation: Federica Valente, Constantin Stefanidis, Ana Roussoulières

Methodology: Federica Valente, Ana Roussoulières

Project administration: Ana Roussoulières

Resources: Constantin Stefanidis, Ana Roussoulières

Supervision: Constantin Stefanidis, Jean-Luc Vachiéry, Céline Dewachter, Ana Roussoulières

Writing - original draft: Federica Valente, Ana Roussoulières

Writing - review \& editing: Constantin Stefanidis, Jean-Luc Vachiéry, Céline Dewachter, Edgard Engelman, Frédéric Vanden Eynden, Ana Roussoulières

\section{Acknwoledgements}

AR had full access to all of the data in the study and takes responsibility for the integrity of the data and the accuracy of the data analysis. FV and AR designed the study, interpreted data and drafted the manuscript. FV collected and analyzed data. EE performed statistical analysis. CS, JLV, CD, EE, FVE have made substantial contributions to conception and design and interpretation of data, and revised the 
article critically for important intellectual content. All authors have provided final approval of the version to be published.

\section{References}

1. Ponikowski P, Voors A. et al. 2016 ESC Guidelines for the diagnosis and treatment of acute and chronic heart failure. European Heart Journal (2016) 37, 2129-2200.

2. Crespo-Leiro M, Metra M, Lund LH, Milicic D, Costanzo MR et al. Advanced heart failure: a position statement of the Heart Failure Association of the European Society of Cardiology. Eur J Heart Fail. 2018 Nov;20(11):1505-1535.

3. Banner NR, Bonser RS, Clark AL, Clark S et al. UK guidelines for referral and assessment of adults for heart transplantation. Heart 2011;97:1520-1527.

4. Mehra M, Kobashigawa J, Starling R, Russell S et al. Listing criteria for heart transplantation: International Society for Heart and Lung Transplantation guidelines for the care of cardiac transplant candidates-2006. J Heart Lung Transplant 2006;25:1024-1042.

5. Rahmel A, ed. Eurotransplant International Foundation Annual Report 2013. Leiden, The Netherlands: CIP-Gegevens Koninklijke Bibliotheek, 2013.

6. Trivedi JR, Cheng A, Singh R, Williams ML, Slaughter MS. Survival on the heart transplant waiting list: impact of continuous flow left ventricular assist device as bridge to transplant. Ann Thorac Surg 2014;98:830-834.

7. Miller LW, Pagani FD, Russell SD, John R, Boyle AJ, et al. Use of a continuous-flow device in patients awaiting heart transplantation. N Engl J Med 2007;357:885-896.

8. Teuteberg JJ, Slaughter MS, Rogers JG, McGee EC, Pagani FD, et al. The HVAD left ventricular assist device: risk factors for neurological events and risk mitigation strategies. JACC Heart Fail 2015;3:818-828.

9. Kirklin JK, Naftel DC, Pagani FD, Kormos RL, Stevenson LW, Blume ED, Myers SL, Miller MA, Baldwin JT, Young JB. Eighth annual INTERMACS report: Special focus on framing the impact of adverse events. The Journal of Heart and Lung Transplantation 2017 36, 1080-1086.

10. Slaughter MS, Rogers JG, Milano CA, Russell SD, et al. HeartMate II Investigators. Advanced heart failure treated with continuous-flow left ventricular assist device. N Engl J Med. 2009;361:22412251.

11. Pagani FD, Miller LW, Russell SD, Aaronson KD, John R, Boyle AJ, et al. HeartMate II Investigators. Extended mechanical circulatory support with a continuous-flow rotary left ventricular assist device. J Am Coll Cardiol. 2009;54:312-321.

12. Matthews JC, Koelling TM, Pagani FD, Aaronson KD. The right ventricular failure risk score a preoperative tool for assessing the risk of right ventricular failure in left ventricular assist device candidates. J Am Coll Cardiol. 2008;51:2163-2172. 
13. Kormos RL, Teuteberg JJ, Pagani FD, Russell SD, John R, Miller LW, et al. Right ventricular failure in patients with the Heartmate II continuous-flow left ventricular assist device: incidence, risk factors, and effect on outcomes. J Thorac Cardiovasc Surg 2010;139:1316-24.

14. Atluri P, Goldstone AB, Fairman AS, MacArthur JW, Shudo Y, Cohen JE, et al. Predicting right ventricular failure in the modern, continuous flow left ventricular assist device era. Ann Thorac Surg 2013;96:857-63.

15. Baumwol J, Macdonald PS, Keogh AM, et al. Right heart failure and "failure to thrive" after left ventricular assist device: clinical predictors and outcomes. J Heart Lung Transplant 2011;30:888-95.

16. Interagency Registry for Mechanically Assisted Circulatory Support (INTERMACS). Appendix A: Adverse event definitions: adult and pediatric patients(2013). Available at http://www.uab.edu/medicine/ intermacs/appendices-4-0/appendix-a-4-0. Accessed September 23, 2014.

17. Holman WL, Acharya D, Siric F, Loyaga-Rendon RY. Assessment and management of right ventricular failure in left ventricular assist device patients. Circ J. 2015;79:478-486. doi: 10.1253/circj.CJ-150093.

18. Dang NC, Topkara VK, Mercando M, Kay J, Kruger KH, Aboodi MS, et al. Right heart failure after left ventricular assist device implantation in patients with chronic congestive heart failure. J Heart Lung Transplant 2006;25:1-6.

19. Lampert B.C., Teuteberg J.J. Right ventricular failure after left ventricular assist devices. J Heart Lung Transplant. 2015; 34: 1123-1130

20. Drakos SG, Janicki L, Horne BD, Kfoury AG, Reid BB, Clayson S, et al. Risk factors predictive of right ventricular failure after left ventricular assist device implantation. Am J Cardiol 2010;105:1030-5.

21. Matthews J, Koelling T, Pagani D, Aaronson K. The Right Ventricular Failure Risk Score: A PreOperative Tool for Assessing the Risk of Right Ventricular Failure in Left Ventricular Assist Device Candidates. J Am Coll Cardiol. 2008 June 3; 51(22): 2163-2172.

22. Wang $Y$, Simon MA, Bonde P, Harris BU, Teuteberg JJ, Kormos RL, et al. Decision tree for adjuvant right ventricular support in patients receiving a left ventricular assist device. J Heart Lung Transplant 2012;31:140-9.

23. Soliman O.I. et al. Derivation and validation of a novel right-sided heart failure model after implantation of continuous flow left ventricular assist devices: the EuroMACS (European Registry for Patients with Mechanical Circulatory Support) right-sided heart failure risk score. Circulation. 2018;137:891-906.

24. Peters A, et al. Comparative Analysis of Established Risk Scores and Novel Hemodynamic Metrics. Journal of Cardiac Failure Vol. 25 No. 8 August 2019. J Card Fail. 2019 Aug;25(8):620-628.

25. Fitzpatrick JF, Frederick JR, Hsu VM, et al. Risk score derived from preoperative data analysis predicts the need for biventricular mechanical circulatory support. J Heart Lung Transplant 2008; 27:1286-92 
26. Bellavia D, lacovoni A, Scardulla C. et al. Prediction of right ventricular failure after ventricular assist device implant: systematic review and meta-analysis of observational studies. European Journal of Heart Failure (2017) 19, 926-946.

27. Swan HJ, Ganz W, Forrester J, et al. Catheterization of the heart in man with use of a flow directed balloon tipped catheter. N Engl J Med 1970; 283:447.

28. Kovacs G, Avian A, Pienn M, Naeije R, Olschewski H. Reading pulmonary vascular pressure tracings. How to handle the problems of zero leveling and respiratory swings. Am J Respir Crit Care Med 2014;190:252-257.

29. Harjola VP, Mebazaa A. et al. Contemporary management of acute right ventricular failure: a statement from the Heart Failure Association and the Working Group on Pulmonary Circulation and Right Ventricular Function of the European Society of Cardiology. Eur J Heart Fail. 2016 Mar;18(3):226-41.

30. Feldman D, Pamboukian SV, Teuteberg JJ, et al. The 2013 International Society for Heart and Lung Transplantation Guidelines for mechanical circulatory support: Executive summary. J Heart Lung Transplant 2013;32:157-87.

31. John R, Bolye A. Preoperative patient optimization for mechanical circulatory support. In: Kormos RL, Miller LW, editors. Mechanical circulatory support. Philadelphia:Elsevier;2011:94.

32. Argenziano M, Choudhri AF, Moazami N. Randomized, double-blind trial of inhaled nitric oxide in LVAD recipients with pulmonary hypertension. Ann Thorac Surg 1998;65:340-5.

33. Hillgaard TK et al. Levosimendan prevents pressure-overload-induced right ventricular failure. J Cardiovasc Pharmacol. 2016;67:275-282.

34. Kirklin JK, Naftel DC, Kormos RL, et al. Fifth INTERMACS annual report: risk factor analysis from more than 6,000 mechanical circulatory support patients. J Heart Lung Transplant 2013;32:141-56.

35. Kapur NK, Esposito M. et al. Mechanical Circulatory Support Devices for Acute Right Ventricular Failure. Circulation. 2017;136:314-326.

36. Fitzpatrick JR, et al.Early planned institution of biventricular mechanical circulatory support results in improved outcomes compared with delayed conversion of a left ventricular assist device to a biventricular assist device.JThorac CardiovascularSurg2009;137:971-7.

37. Morgan JA,et al.Is severe right ventricular failure in left ventricular assist device recipients a risk factor for unsuccessful bridging to transplant and post-transplant mortality. Ann Thorac Surg 2004;77:859-63.

\section{Figures}




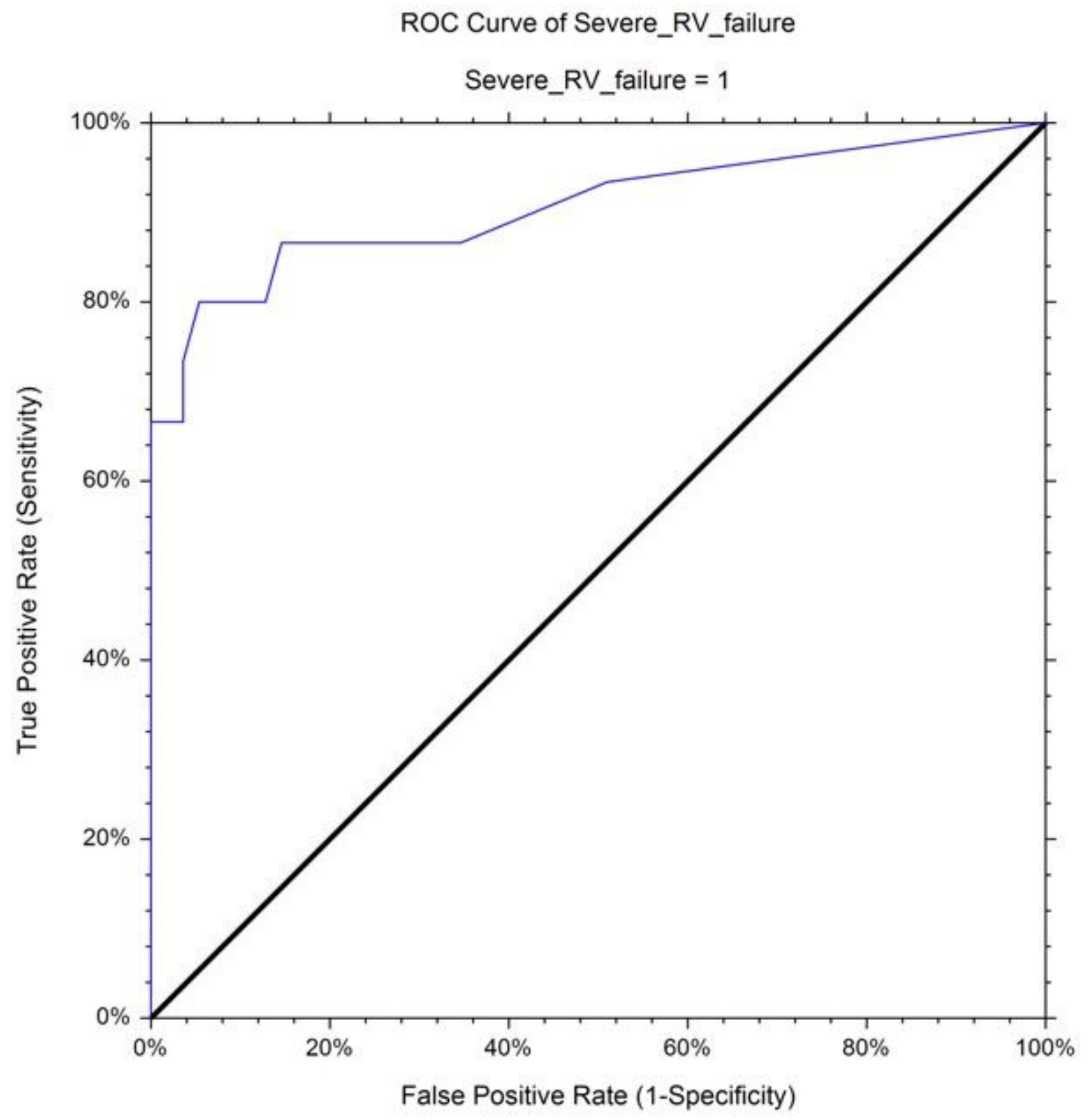

\section{Figure 1}

Receiver operating characteristic curve (ROC) derived from the logistic regression model for severe right ventricular failure. Area under ROC Curve for Severe RV failure $=0.91-95 \%$ Cl: $0.76-0.96$ Area under ROC Curve for No Severe RV failure $=0.89-95 \%$ Cl: 0.70- 0.96 


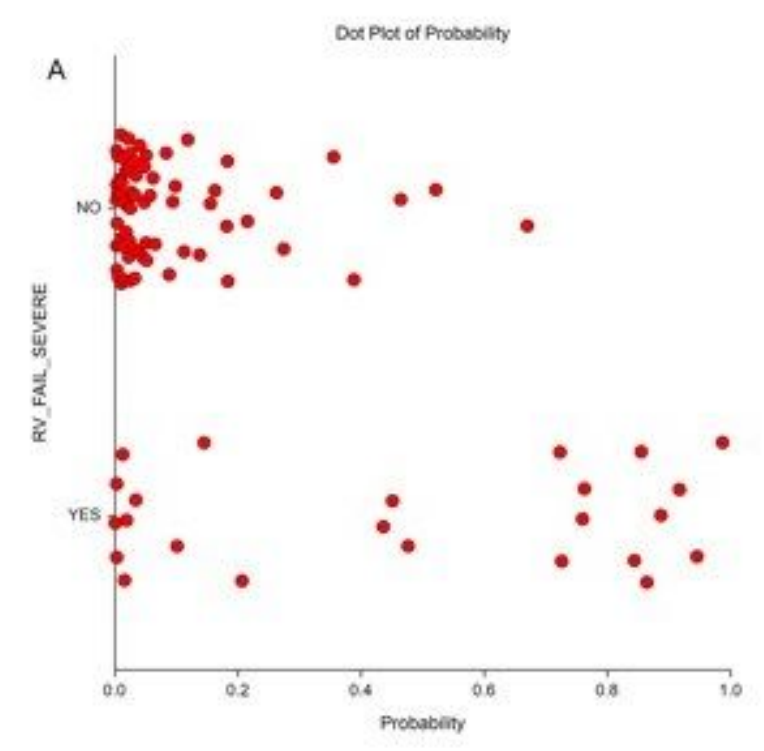

B
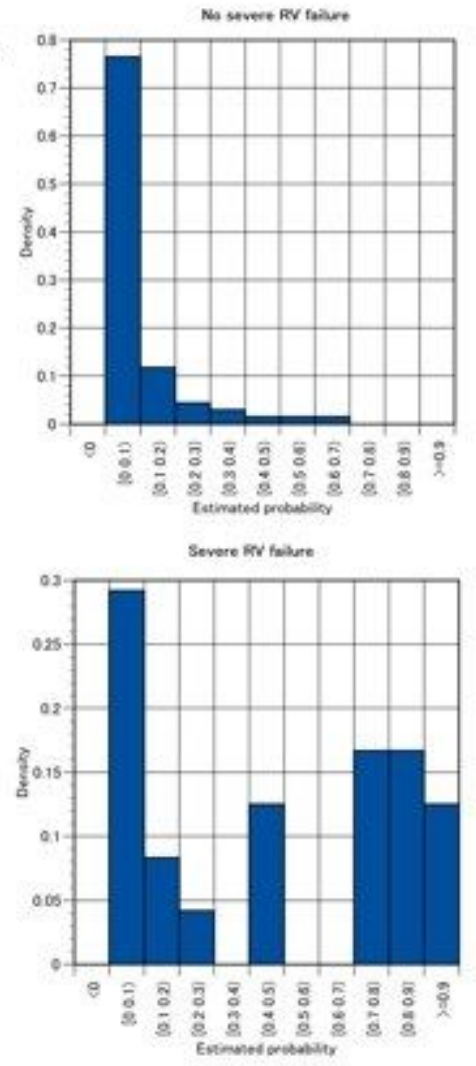

Figure 2

Panel A: Plot of jittered outcome versus estimated probabilities from the fitted model: $-11.48+2.98$ * RV / $\mathrm{LV}+6.17$ * RAP / PCWP + 1.93 * inotropes + 1.73 * (INTERMACS_GROUP="1-3") RV = right ventricle diameter $\mathrm{LV}=$ left ventricle diameter RAP = right atrial pressure $\mathrm{PCWP}=$ pulmonary capillary wedge pressure inotropes $=$ number of inotropic drugs Panel B: Histogram of estimated probabilities from the fitted model for the occurrence or not of severe right ventricular failure. Derived from the same model as in Panel A 


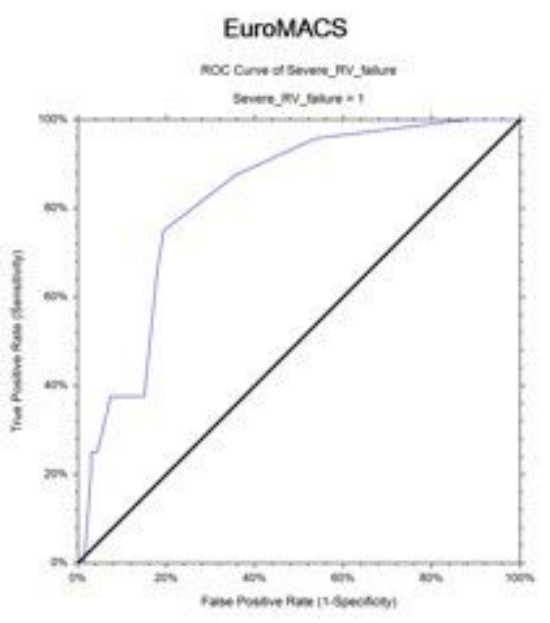

Area under ROC Curve for Severe RV failure: $0.82-95 \%$ Ct: $0.68-0.90$

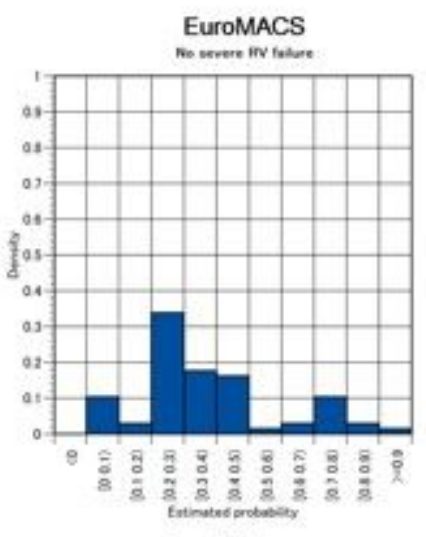

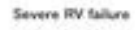

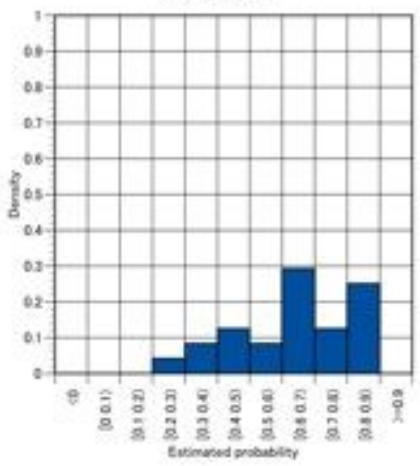

Michigan risk score

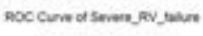

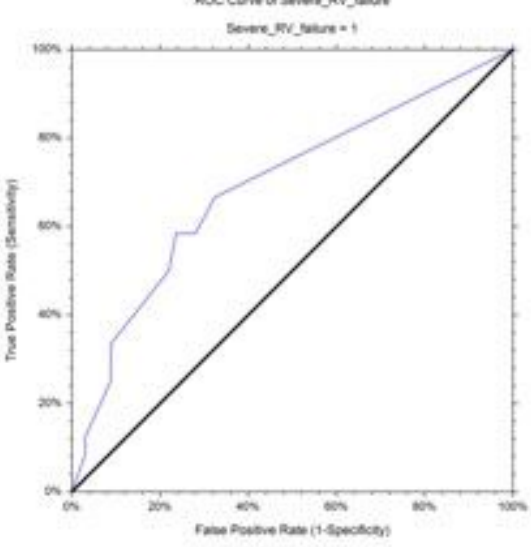

Area under ROC Curve for Severe RV falure: $0.69-95 \% \mathrm{Cl}: 0.54-0.80$

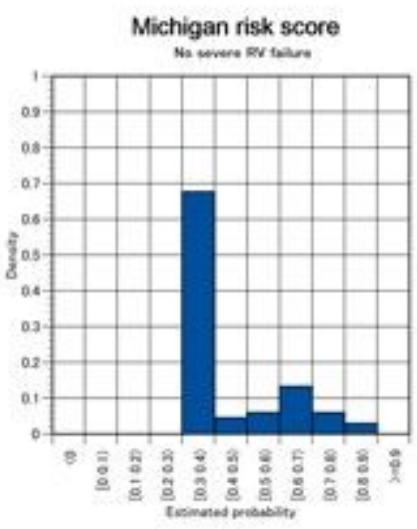

Serene Hiv talase

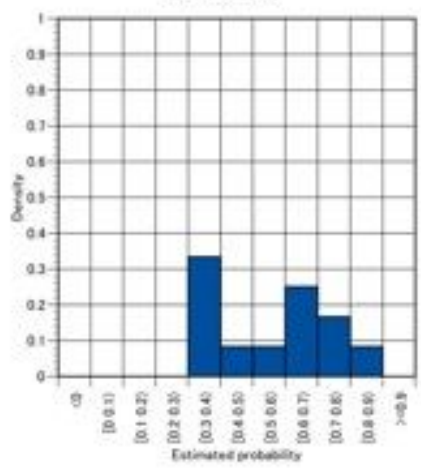

CRITT score

noc Carmes the

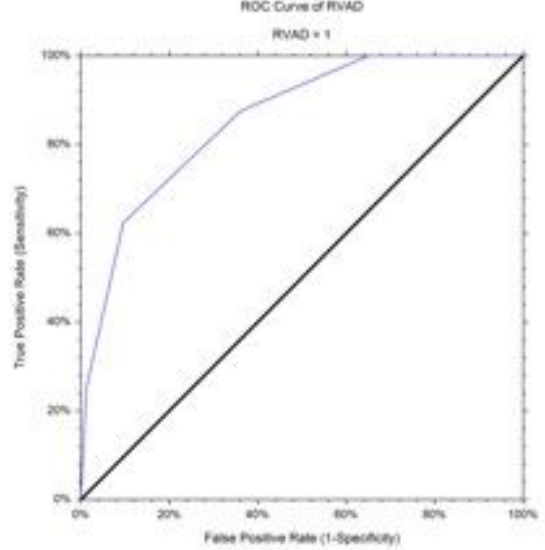

Area under ROC Curve for RVAD placement:

$0.86-95 \%$ Ct: $0.72-0.93$
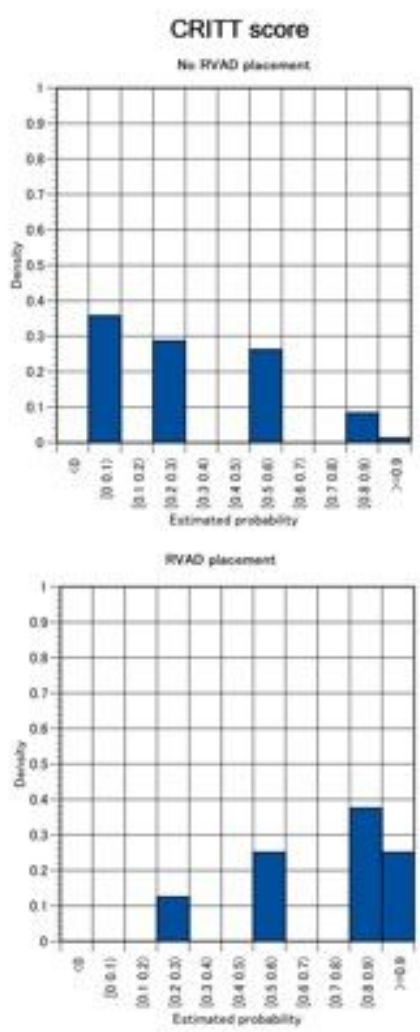

Figure 3

Receiver operating characteristic curves (ROC) derived from the logistic regression models for severe right ventricular failure (EUROMACS and Michigan risk score) or for right ventricular assist device placement (CRITT score) from their respective scores in our population, and the associated histograms of estimated probabilities from the fitted models.

\section{Supplementary Files}

This is a list of supplementary files associated with this preprint. Click to download. 
- supplementaltable1.doc

Page 28/28 